\title{
Thermodynamic Properties of Small Magnetic Particles
}

\author{
Vanessa Souza Leite and Wagner Figueiredo \\ Departamento de Física - Universidade Federal de Santa Catarina \\ 88040-900, Florianópolis, SC - Brazil
}

\section{Received on 7 September, 2005}

\begin{abstract}
We investigate the equilibrium magnetic properties of a simple cubic small ferromagnetic particle under an external magnetic field. Although the particle is small, it can not be considered as a single-domain unit. The magnetic moments are represented by unitary spin vectors and we consider ferromagnetic interactions between nearest-neighbor spins. The coupling between spins is given in terms of the classical Heisenberg Hamiltonian and we also include a Zeeman contribution and a single-ion uniaxial anisotropy. The size of the particle changes from one to twelve lattice spacings. We employ in our study mean-filed calculations and Monte Carlo simulations. The magnetization and susceptibility curves as a function of temperature show that an uniaxial anisotropy can mimic a ferromagnetic or an antiferromagnetic coupling, depending on the angle between the external magnetic field and the easy axis.
\end{abstract}

Keywords: Magnetismo; Finite systems; Monte Carlo simulations

\section{INTRODUCTION}

The development of new techniques that opened the doors to see the details of the matter at the nanoscale, also launched the area of nanomagnetism, where we look at the behavior of small magnetic particles. This field of research has shown to be fruitfull not only from the fundamental point of view, but also for the possible technological applications [1-4]. In this way, many investigations have been developed experimentally $[5,6]$, analytically [7-10], and in computer simulations [9-14] in order to increase our knowledge about these small systems.

In the case of a small magnetic particle, its size can play a crucial role on its behavior. In particular, sufficiently small particles become single-domain particles in which all their atomic moments rotate coherently in the presence of an external magnetic field [8]. On the other hand, when the size of the particles is increased, the single domain configuration becomes unfavorable and the particle assumes a many-domain configuration [13].

We investigate in this work the equilibrium magnetic properties of a small ferromagnetic particle that is large enough to be considered as a multi-domain particle. We assume three different types of energy contributions for this particle: the exchange ferromagnetic interactions between the nearestneighbor spins, the Zeeman energy, due to presence of an external magnetic field and the single-ion uniaxial anisotropy energy. To study the magnetic properties of the particle we employ both mean-field calculations and Monte Carlo simulations. As we show below, the results obtained from the analysis of the magnetization and susceptibility curves as a function of temperature show that the effect of the anisotropy on the magnetic properties of the particle depends crucially on the angle between the easy axis and the magnetic field. Particularly, although the coupling between nearest-neighboring spins is ferromagnetic, an easy axis perpendicular to the field can produce an overall antiferromagnetic behavior, as if the coupling between nearest-neighboring spins was antiferromagnetic.
In the following, we present in Section II the model and the equations for the magnetization in the mean field approximation. In Section III, we describe the Monte Carlo simulations. Next, in Section IV, we present our results and conclusions.

\section{THE MODEL}

The magnetic moments of the particle are distributed on concentric spherical layers which are inscribed into a simple cubic structure. Each site of the lattice that belongs to a given spherical layer represents a magnetic moment of the particle. The magnetic moments are vectors of unitary magnitude, $\left|\vec{S}_{i}\right|=1$. These magnetic moments interact through the classical Heisenberg Hamiltonian:

$$
\mathcal{H}=-\frac{J}{2} \sum_{i}^{N} \sum_{j}^{q} \vec{S}_{i} \cdot \vec{S}_{j}-\sum_{i}^{N} \vec{H} \cdot \vec{S}_{i}-\sum_{i}^{N} k\left(\hat{e}_{k} \cdot \vec{S}_{i}\right)^{2},
$$

where $N$ is the number of spins in the particle, $q$ is the coordination number of the magnetic moments $(q=6$ for the internal spins), $J$ is the ferromagnetic exchange coupling, $\vec{H}$ represents the external magnetic field, $k$ is the anisotropy constant and the unitary vector $\hat{e}_{k}$ fixes the direction of the easy axis of the particle.

We obtain the equilibrium magnetic properties of the particle through the mean-field approximation by employing the Bogoliubov's Inequality [15]. In this way, the exact free energy $G$ of the system, which is described by the Hamiltonian $\mathcal{H}$, satisfies the inequality

$$
G \leq G_{0}+<\mathcal{H}-\mathcal{H}_{0}>_{0} \equiv \Phi
$$

where $G_{0}$ is a trial free energy, which is calculated through a Hamiltonian $\mathcal{H}_{0}$. $\mathcal{H}_{0}$ is an approximation to the exact Hamiltonian $\mathcal{H}$. 
In the mean-field scheme, we write $\mathcal{H}_{0}$ as a sum of noninteracting spins, weighted by a set of variational parameters. Therefore, to obtain the free energy of the particle in the mean-field approximation, $G_{M F}$, we need to minimize $\Phi$ relative to the variational parameters.

In the following we consider three different cases in the study of the small ferromagnetic particle: zero anisotropy case, the easy axis of the particle is parallel to the external magnetic field, and the easy axis of the particle is perpendicular to the external magnetic field. To simplify, we take the external magnetic field in the $z$-direction.

In the first case, where the particle is isotropic $(k=0)$, we take the following trial Hamiltonian $\mathcal{H}_{0}$ :

$$
\mathcal{H}_{0}=-\sum_{i}^{N} n_{i} S_{i}^{z}
$$

where the $n_{i}$ are the variational parameters used to minimize $\Phi$.

After some calculations, we obtain the equation of state of each magnetic moment of the particle

$m_{i}=<S_{i}^{z}>=\operatorname{coth}\left[\beta\left(H+\frac{J}{2} \sum_{j}^{q} m_{j}\right)\right]-\frac{1}{\left[\beta\left(H+\frac{J}{2} \sum_{j}^{q} m_{j}\right)\right]}$,

and $\left\langle S_{i}{ }^{x}\right\rangle=\left\langle S_{i}^{y}\right\rangle=0$. Therefore, the magnetization of the particle can be written as

$$
m(T, H)=\frac{1}{N} \sum_{i}^{N} m_{i}
$$

In the case in which the easy axis is parallel to the external magnetic field, we choose the following trial Hamiltonian

$$
\mathcal{H}_{0}=-\sum_{i}^{N} n_{i} S_{i}^{z}-\sum_{i}^{N} p_{i} S_{i}^{z 2}
$$

where $n_{i}$ and $p_{i}$ are the variational parameters of the problem.

Therefore, the equation of state for each spin of the particle is

$m_{i}=<S_{i}^{z}>=\frac{\int_{-1}^{1} \int_{-b}^{b} \int_{-a}^{a} S_{i}^{z} e^{\beta\left[\left(H+\frac{J}{2} \sum_{j}^{q} m_{j}\right) S_{i}^{z}+k S_{i}^{\left.z^{2}\right]}\right.} d S_{i}^{x} d S_{i}^{y} d S_{i}^{z}}{\int_{-1}^{1} \int_{-b}^{b} \int_{-a}^{a} e^{\beta\left[\left(H+\frac{J}{2} \sum_{j}^{q} m_{j}\right) S_{i}^{z}+k S_{i}^{z 2}\right]} d S_{i}^{x} d S_{i}^{y} d S_{i}^{z}}$,

with $\left\langle S_{i}^{x}>=<S_{i}^{y}>=0, a=\sqrt{1-S_{i}^{y 2}-S_{i}^{z^{2}}}\right.$ and $b=$ $\sqrt{1-S_{i}^{z^{2}}}$

Finally, when the easy axis of the particle is perpendicular to the external magnetic field, the trial Hamiltonian $\mathcal{H}_{0}$ is

$$
\mathcal{H}_{0}=-\sum_{i}^{N} n_{i} S_{i}^{z}-\sum_{i}^{N} q_{i} S_{i}^{x}-\sum_{i}^{N} p_{i} S_{i}^{x 2}
$$

where $n_{i}, q_{i}$ and $p_{i}$ are the variational parameters.

The equation of state for each one of the magnetic moments of the particle takes the form

$$
m_{i}=m_{i}^{z}=<S_{i}^{z}>=\frac{\int_{-1}^{1} \int_{-b}^{b} \int_{-a}^{a} S_{i}^{z} e^{\beta\left[\left(H+\frac{J}{2} \sum_{j}^{q} m_{j}^{z}\right) S_{i}^{z}+\left(\frac{J}{2} \sum_{j}^{q} m_{j}^{x}\right) S_{i}^{x}+k S_{i}^{x}\right]} d S_{i}^{x} d S_{i}^{y} d S_{i}^{z}}{\int_{-1}^{1} \int_{-b}^{b} \int_{-a}^{a} e^{\beta\left[\left(H+\frac{J}{2} \sum_{j}^{q} m_{j}^{z}\right) S_{i}^{z}+\left(\frac{J}{2} \sum_{j}^{q} m_{j}^{x}\right) S_{i}^{x}+k S_{i}^{x 2}\right]} d S_{i}^{x} d S_{i}^{y} d S_{i}^{z}},
$$

where

$$
m_{i}^{x}=<S_{i}^{x}>=\frac{\int_{-1}^{1} \int_{-b}^{b} \int_{-a}^{a} S_{i}^{x} e^{\beta\left[\left(H+\frac{J}{2} \sum_{j}^{q} m_{j}^{z}\right) S_{i}^{z}+\left(\frac{J}{2} \sum_{j}^{q} m_{j}^{x}\right) S_{i}^{x}+k S_{i}^{x 2}\right]} d S_{i}^{x} d S_{i}^{y} d S_{i}^{z}}{\int_{-1}^{1} \int_{-b}^{b} \int_{-a}^{a} e^{\beta\left[\left(H+\frac{J}{2} \sum_{j}^{q} m_{j}^{z}\right) S_{i}^{z}+\left(\frac{J}{2} \sum_{j}^{q} m_{j}^{x}\right) S_{i}^{x}+k S_{i}^{x 2}\right]} d S_{i}^{x} d S_{i}^{y} d S_{i}^{z}}
$$

with $<S_{i}^{y}>=0, a=\sqrt{1-S_{i}{ }^{y 2}-S_{i}^{z 2}}$ and $b=\sqrt{1-S_{i}^{z 2}}$. The magnetization of the particle is obtained from the equation

$$
m(T, H)=\frac{1}{N} \sum_{i}^{N} m_{i}^{z}
$$

For each one of the three cases considered, we must solve a set of coupled equations for the magnetic moments of the par- ticle, and from these, finding the average magnetization as a function of temperature and magnitude of the anisotropy. The number of equations $\left(N_{E}\right)$ is smaller than the number of spins $\left(N_{S}\right)$ in the particle, due to the symmetry conditions. For instance, for a particle with only a single shell, we have $N_{S}=7$ and $N_{E}=2$; for a particle with six shells, we have $N_{S}=925$ and $N_{E}=95$, and for a particle with twelve shells, we have $N_{S}=7133$ and $N_{E}=579$. To solve the set of coupled equations, we used one of the routines presented in the Numerical 
Recipes book [16].

\section{MONTE CARLO SIMULATIONS}

The ferromagnetic particle was simulated by employing the Metropolis algorithm [17, 18]. In each Monte Carlo step (MCs), we performed $N$ ( $N$ is the number of spins of the particle) trials to flip randomly one magnetic moment of the particle. To calculate the average magnetic properties of the particle we took in general $5 \times 10^{4} M C s$, where the first $2 \times 10^{4}$ $M C s$ were discarded due to the thermalization process.

In our algorithm we calculate the average magnetization of the particle, as well as, its components along the $x, y$ and $z$ directions as a function of temperature and strength of anisotropy. These mean values are obtained firstly by considering the mean value among the magnetic moments in the particle:

$$
\begin{gathered}
m^{x}=\frac{1}{N} \sum_{i=1}^{N} S_{i}^{x}, \\
m^{y}=\frac{1}{N} \sum_{i=1}^{N} S_{i}^{y}, \\
m^{z}=\frac{1}{N} \sum_{i=1}^{N} S_{i}^{z}, \\
m_{t o t}=\sqrt{m^{x 2}+m^{y 2}+m^{z 2}},
\end{gathered}
$$

in each $M C s$ after the thermalization process. In the following, the averages are taken considering the different Monte Carlo steps, where we obtain $\left\langle m^{x}\right\rangle,\left\langle m^{y}\right\rangle,\left\langle m^{z}\right\rangle$ and $\left\langle m_{t o t}\right\rangle$, for each value of temperature and external magnetic field.

We also calculate the fluctuation associated with the magnetization of the particle

$$
\left.\left.\Delta m=\left|<m_{t o t}^{2}\right\rangle-<m_{t o t}\right\rangle \cdot<m_{t o t}\right\rangle \mid .
$$

The results obtained from the Monte Carlo simulations are shown in the next section.

\section{RESULTS AND CONCLUSIONS}

Let us start our analysis by the case where there is no anisotropy. In Fig.1 we present the typical curve for the magnetization of a small ferromagnetic particle obtained through the Monte Carlo simulations. In this figure we have a particle with six shells and we show its magnetization (Fig.1(a)) and the corresponding fluctuations (Fig.1(b)) as a function of temperature, when the external magnetic field is zero. As we note, these curves characterize a ferromagnetic behavior with a critical temperature $T_{c}=(1.29 \pm 0.02) J / k_{B}$. As we can see in Fig.2, the critical temperature (actually the pseudocritical temperature [10]) increases with the size of the particle $[10,19]$ up to reach the thermodynamic limit. In our case this limit is easily found for a particle with nine shells, where we obtain $T_{c}=(1.44 \pm 0.02) \mathrm{J} / k_{B}$, which is very close to the value obtained for a ferromagnetic system [20]. The same qualitative behavior is also seen in the mean-field approach.

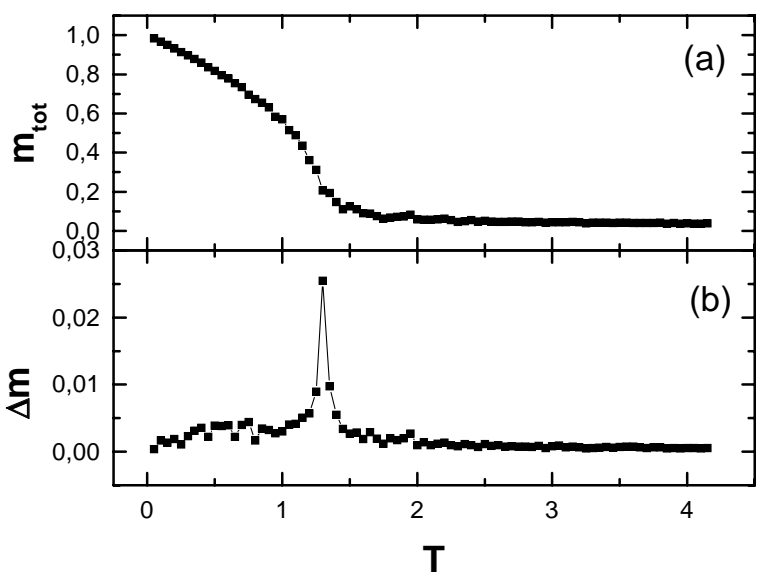

FIG. 1: Typical magnetization curve obtained by Monte Carlo simulations for an isotropic particle: $(a)$ average magnetization of the particle and $(b)$ fluctuation of the magnetization, when the external magnetic field goes to zero. Particle with six shells, for which we have $T_{c}=(1.29 \pm 0.02) J / k_{B}$. Temperature is in units of $J / k_{B}$.

When the particle has an easy axis parallel to the external magnetic field, the same typical curves as presented in Fig.1, which represent a ferromagnetic behavior, appear. In Fig.3 we plot the critical temperature of the particle as function of its size, obtained from Monte Carlo simulations, for different values of the anisotropy constant. As could be expected, the critical temperature increases with the value of the anisotropy constant, that is, it becomes more difficult to demagnetize the particle for large values of the anisotropy.

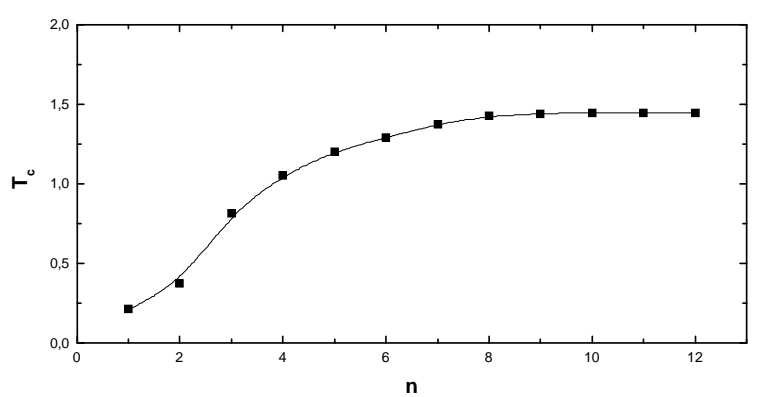

FIG. 2: Critical temperature as a function of the size of the particle obtained by Monte Carlo simulations for an isotropic particle. Temperature is in units of $J / k_{B}$ and $n$ is the number of shells of the particle.

Finally, when the particle has an easy axis perpendicular to the external magnetic field, two distinct behaviors are seen 


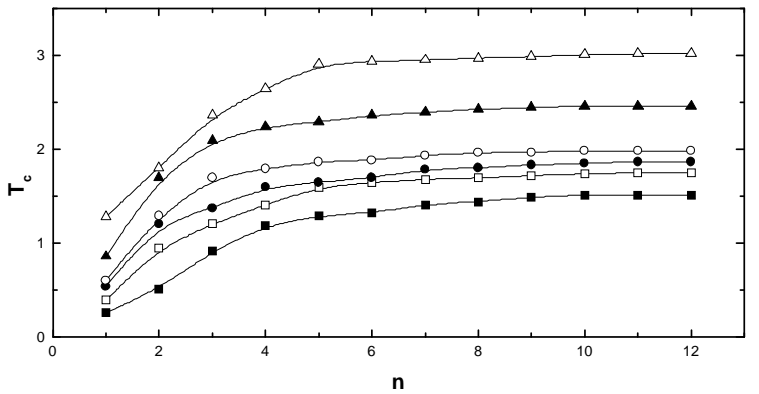

FIG. 3: Critical temperature as a function of the size of the particle for different values of the magnitude of the anisotropy obtained through Monte Carlo simulations. The particle has the easy axis parallel to the external magnetic field. From bottom to top: $k=0.1 \mathrm{~J}$ $(T c=1.50), k=0.5 J(T c=1.75), k=1.0 J(T c=1.87), k=2.0 J$ $(T c=1.98), k=5.0 J(T c=2.46)$ and $k=10.0 J(T c=3.02) . T_{c}$ is the temperature in the thermodynamic limit. Temperature is in units of $J / k_{B}$ and $n$ is the number of shells of the particle.

both in mean-field calculations and Monte Carlo simulations. The usual ferromagnetic behavior is seen for low values of the anisotropy constant. On the other hand, for large values of the anisotropy, the particle presents an antiferromagnetic behavior. The magnitude of the anisotropy constant, for which the particle changes its behavior from ferromagnetic to antiferromagnetic is $k=3.5 \mathrm{~J}$ in the mean-field approximation and $k=3.4 \mathrm{~J}$ in the Monte Carlo simulations.

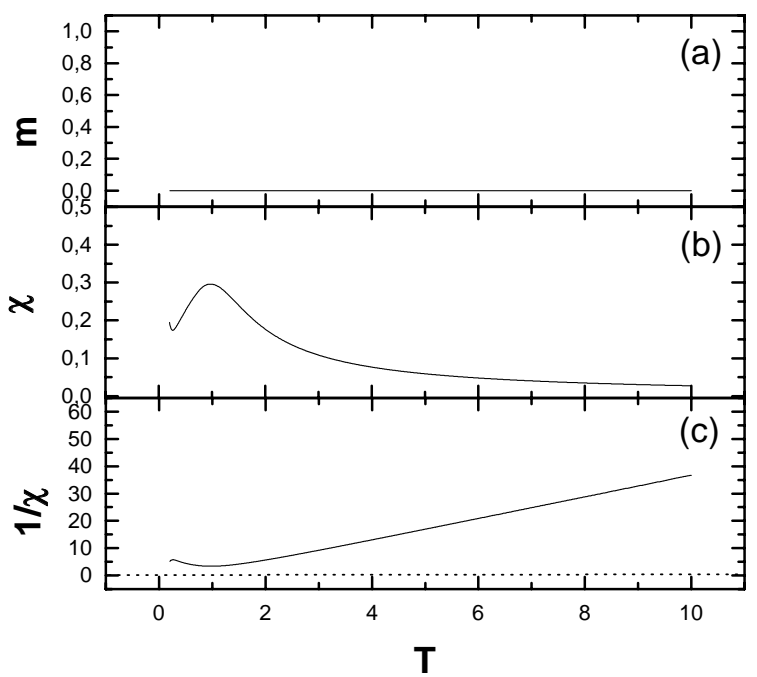

FIG. 4: Typical magnetization curve for the case where the easy axis of the particle is perpendicular to the external magnetic field in the mean-field approach. (a) average magnetization of the particle, $(b)$ susceptibility and $(c)$ inverse of the susceptibility, when the external magnetic field goes to zero. Particle with six shells, $k=5 J$, and $T_{N}=1.02 J / k_{B}$. Temperature is in units of $J / k_{B}$.

For values of the anisotropy larger than these quoted above, the typical magnetization curve and the corresponding fluctuations are displayed in Figs.4 and 5, in the mean-field approach and Monte Carlo simulations, respectively. In both figures we considered a particle with six shells and we took $k=5.0 \mathrm{~J}$.

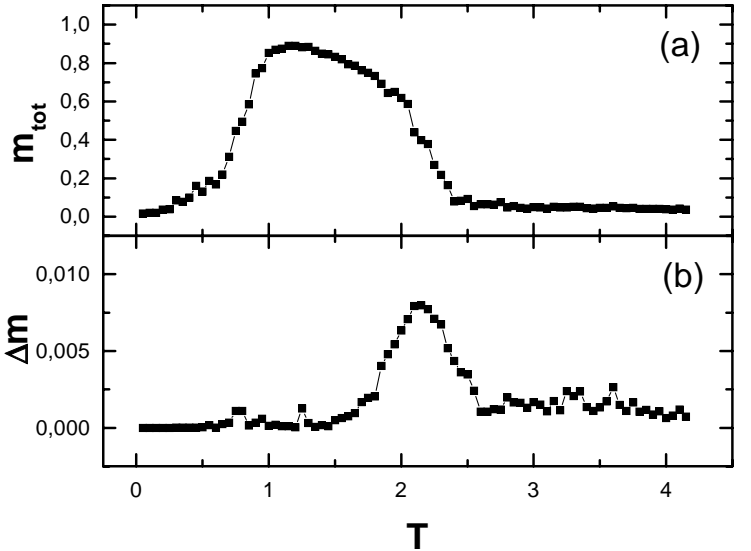

FIG. 5: Typical magnetization curve obtained from Monte Carlo simulations for a particle with its easy axis perpendicular to the external magnetic field. (a) average magnetization of the particle and (b) fluctuations of the magnetization, when the external magnetic field goes to zero. We have a particle with six shells, $k=5.0 \mathrm{~J}$, and $T_{N}=(2.20 \pm 0.02) J / k_{B}$. Temperature is in units of $J / k_{B}$.

In the mean-field case, the magnetization of the particle coincides with the component of total magnetization along the $z$ direction. Therefore, we find a zero magnetization for all values of temperature, once the magnetization in the $z$ direction vanishes at zero external field. On the other hand, in the Monte Carlo calculations, the total magnetization of the particle is obtained along the $x$ direction. In this way, a broad maximum appears in the magnetization curve as a function of temperature. Microscopically, looking at the spin configurations, we clearly see an antiferromagnetic arrangement of the spins for a temperature less than that of the maximum. Besides the magnetization, we verify in both approaches that the susceptibility does not diverge for any value of temperature, but it also exhibits a maximum at a given temperature. This kind of behavior is characteristic of antiferromagnetic systems, and the temperature in which occurs the maximum determines the Neél temperature. The Neél temperature corresponds to the temperature where the system changes its behavior from antiferromagnetic to paramagnetic [21].

To summarize, we have studied a small ferromagnetic particle taking into account the relative orientation of its easy axis to the external magnetic field. If the easy axis is parallel to the magnetic field the particle behaves as a ferromagnetic system, and the effect of the anisotropy is to increase the critical temperature. On the other hand, when the anisotropy axis is perpendicular to the external magnetic field, the magnetic behavior of the particle depends on the magnitude of the anisotropy. For low values of this parameter, the particle continues exhibiting a ferromagnetic behavior, but for high values of anisotropy, the particle presents an overall antiferromagnetic behavior. 


\section{Acknowledgments}

The authors acknowledge the financial support by the Brazilian agencies CNPq and FUNCITEC.

[1] D. Gatteshi, O. Kahn, J. S. Miller and F. Palacio, Magnetic Molecular Materials (NATO ASI Series, Kluwer Academic, Dordrecht,1991).

[2] Science and Technology of Nanostructured Magnetic Materials, edited by G.C. Hadjipanayis e G.A. Prinz (Plenum Press, New York, 1991).

[3] R. Skomski, J. Phys.: Condens. Matter 15, R841 (2003).

[4] R. W. Chantrell, K. O. 'Grady, Applied Magnetism (Kluwer Academic Publishers, Dordrecht,1994).

[5] R. H. Kodama, A. E. Berkowitz, E. J. McNiff, Jr., and S. Foner, Phys. Rev. Lett. 77, 394 (1996).

[6] W. Wernsdorfer, E. B. Orozco, K. Hasselbach, A. Benoit, B. Barbara, N. Demoncy, A. Loiseau, H. Pascard, and D. Mailly, Phys. Rev. Lett. 78, 1791 (1997).

[7] W. T. Coffey, D. S. F. Crothers, J. L. Dormann, Yu. P. Kalmykov, E. C. Kennedey, and W. Wernsdorfer, Phys. Rev. Lett. 80, 5655 (1998).

[8] P. Vargas, D. Altbir, M. Knobel, and D. Laroze, Europhys. Lett. 58, 603 (2002).

[9] V. S. Leite and W. Figueiredo, Physica A 350, 379 (2005).

[10] V. S. Leite, M. Godoy, and W. Figueiredo, Phys. Rev. B 71, 038509 (2005).

[11] P. A. Rikvold, B. M. Gorman, Annual Reviews of Computa- tional Physics I (World Scientific, Singapore,1994).

[12] H. L. Richards, M. Kolesik, P. A. Lindgard, P. A. Rikvold, and M. A. Novotny, Phys. Rev. B 55, 11521 (1997).

[13] D. Hinzke and U. Nowak, Phys. Rev. B 58, 265 (1998).

[14] K. N. Trohidou, X. Zianni, and J. A. Blackman, J. Appl. Phys. 84, 2795 (1998).

[15] H. Falk, Am. J. of Phys. 38, 858 (1970).

[16] W. H. Press, S. A. Teukolsky, W. T. Vetterling, and B. P. Flannery, Numerical Recipes in Fortran 77: The Art of Scientific Computing, 2nd edition (Cambridge University Press, UK, 1997).

[17] K. Binder, Monte Carlo Methods in Statistical Physics (Springer, Berlin, 1979).

[18] D. P. Landau and K. Binder, A Guide to Monte Carlo Simulations in Statistical Physics (Cambridge University Press, UK, 2000).

[19] O. Iglesias and A. Labarta, Phys. Rev. B 63, 184416 (2001).

[20] K. Chen, A. M. Ferrenberg, and D. P. Landau, Phys. Rev. B 48, 3249 (1993).

[21] C. Kittel, Introduction to Solid State Physics (5th Edition, Wiley, New York, 1996). 